\title{
The Emerging Role of Circular RNAs in Cerebral Vascular Disorders
}

\author{
Wei Liu GaoYan \\ Department of Neurology, Shengjing Hospital of China Medical University, Shenyang, China
}

\section{Keywords}

Circular RNAs · MicroRNAs · Atherosclerosis · Stroke

\begin{abstract}
Background: Circular RNAs (circRNAs) are covalently closed circular noncoding RNAs that are expressed in various life forms. CircRNAs have many characteristics, such as structural stability and tissue-specific expression that contribute to their role as a microRNA (sponge in gene regulation. Summary: Recent evidence suggests that circRNAs play an important role in the pathogenesis of cerebrovascular diseases (CVDs); however, the exact mechanism remains controversial. CircRNAs that are related to CVDs have great clinical significance. Key Messages: The present review provides an overview of the general biology of circRNAs, their relevant regulatory mechanisms, and their role in the pathophysiology of CVDs.

(C) 2021 S. Karger AG, Basel
\end{abstract}

\section{Introduction}

Circular RNAs (circRNAs) are a special type of noncoding RNA molecules that are different from linear RNAs. CircRNAs derive from exon or intron regions of the gene and have been a focus in the field of RNA mo- lecular biology in recent years. CircRNAs form a closedloop structure through covalent bonds that are not easily degraded by exonuclease and are more stable than linear RNAs. Sanger et al. [1] first discovered circRNAs in plant diseases in the 1970s. In 2012, Salzman et al. [2, 3] used RNA sequencing to study cancer-related chromosomal rearrangements and gene fusions. CircRNAs were first reported to exist not only in cancer cells but also in normal human cell lines and mouse brain tissues. The total amount of circRNAs in cells is $\sim 1 \%$ of linear RNAs. The abundance of circRNAs exceeds linear RNAs that correspond to the allele locus $[2,3]$. Accumulating studies have shown that circRNAs are widely present in nervous system tissues and involved in various pathophysiological processes, including inflammation, oxidative stress, mitochondrial dysfunction, and apoptosis $[4,5]$.

In recent decades, cerebral vascular diseases (CVDs) have become a leading cause of death worldwide. They are one of the most important diseases that endangering human health in today's society, the incidence of which is rising as the age of the population increases [6]. Although treatments for CVDs are improving, some patients still have a poor long-term prognosis. Based on the involvement of circRNAs in the pathogenesis and progression of CVDs, such as inflammation, oxidative stress, mitochondrial dysfunction, and apoptosis, molecular targets that are useful for disease prediction and treatment are being 
actively explored. The present review provides an overview of the regulatory mechanisms of circRNAs in CVDs and discusses the role of circRNAs in its pathophysiology, with the goal of opening new perspectives for poststroke brain protection therapy.

\section{Biosynthesis and Classification of CircRNAs}

Four mechanisms are involved in the biosynthesis of circRNAs. First, extron-skipping circularization produces a lariat-driven circularization that contains exons and introns that are then reverse spliced and deleted, and intron sequences are retained to form exonic circRNA (ecircRNA) or exon-intron ecircRNA (EIciRNA) [7]. Second, intron pairing-driven circularization occurs in flanked intron sequences that contain inverted repeats or ALU repeats of pre-RNA. Such intron pairing allows the $5^{\prime}$ splice site of the upstream exon and $3^{\prime}$ splice site of the downstream exon to be close to each other, and the head and tail are joined by reverse splicing to form ecircRNA or EIciRNA [8]. Third, intron lariatdriven circularization involves an intron lariat that is formed by pre-RNA during classic splicing, the intron $5^{\prime}$ splice site of which is enriched with 7 nt GU repeats, and the $3^{\prime}$ branch point is rich in 11 nt $C$ repeats. This special sequence ensures the stability of circRNAs and ultimately forms ciRNA [9]. Fourth, RNA-binding protein-driven circularization is similar to intron-driven circularization. The RNA-binding protein interacts with flanking intron sequences to cause 2 flanking introns to be close to each other, thereby promoting cyclization and joining them by reverse splicing to form ecircRNA or EIciRNA [10].

CircRNAs can derive from any region of the genome, including exons, introns, intergenic regions, untranslated regions, or antisense strands that range in length from a few 100 to several 1,000 nucleotides. Human circRNAs mainly derive from coding genes. According to the gene source of the circRNA molecule, it can be divided into ecircRNA, intron circRNA (icircRNA), EIciRNA, and 4 types of intergenic circRNA (itraRNA). Among these, ecircRNA is the most abundant circRNA, which is mainly located in the cytoplasm, whereas ciRNA and EIciRNA are mainly located in the nucleus $[2,9,11,12]$.

\section{Biological Function and Mechanism of CircRNAs}

CircRNAs have been shown to have the following functions. Nuclear-related functions include transcriptional regulation and the regulation of alternative RNA splicing $[12,13]$. However, most studies have focused on cytoplasm-related functions.

Circular RNAs in Cerebral Vascular Disorders
Regulation of MicroRNAs

CircRNAs contain a large number of microRNA (miRNAs) binding sites, called miRNAs response elements, that can inhibit the binding of miRNAs to their target genes in the cytoplasm, thereby promoting or inhibiting the expression of target genes [14]. CircRNAs can have multiple miRNAs response elements with multiple miRNAs and multiple binding sites for a single miRNAs. This absorption of miRNAs is referred to as a miRNAs sponge effect [15]. A classic study investigated the circRNA sponge of miRNA-7, also known as the antisense transcript of cerebellar degeneration-related protein 1 (Cdr1as), which negatively regulates the expression of miRNA-7 [16]. Cdrlas contains $>70$ miRNA-7 binding sites that are highly expressed in HEK293 cells and can bind up to 20,000 miRNA-7 molecules in each cell. Cdrlas can also bind Argonaute protein and absorb miRNA-7 in brain tissue, thus inhibiting its negative regulation of target genes [11]. In addition to simple inhibition, the interaction between circRNAs and miRNAs is related to the storage, sorting, and localization of miRNAs.

\section{Direct Impact on Gene Expression}

CircRNAs are directly involved in the regulation of gene expression by regulating linear RNA transcription and variable splicing. For example, in an exon hopping model, precursor RNA can be alternatively spliced to form mature linear RNAs while cyclizing to form ecircRNA. Thus, the synthesis of ecircRNA can hinder the synthesis of homologous linear RNA [17]. Additionally, the cyclization and nonlinear splicing of pre-RNA that contains translation initiation sites mean that the formation of circRNAs reduces the formation of RNA and the translation of downstream proteins, which is known as an RNA trap [18].

\section{Encoding Proteins}

Because of their lack of some common characteristics of coding RNAs, circRNAs have been considered noncoding RNAs. However, a study of encephalomyocarditis virus found multiple internal ribosome entry sites in circRNAs, suggesting that circRNAs might also have a protein-encoding function [19]. The m6A modification of circRNAs can promote its own translation, indicating that circRNAs can also express proteins or polypeptides with external stimulation [20].

\section{Regulation of Protein Function}

CircRNAs can indirectly regulate gene expression by binding to some key RNA-binding proteins [21]. Previ- 
Table 1. Overview of the circRNAs expression in the models of CVDs

\begin{tabular}{|c|c|c|c|c|c|c|c|}
\hline CVDs & Model & Method & $\begin{array}{l}\text { CircRNA } \\
\text { alteration }\end{array}$ & CircRNA & Down molecules/genes & $\begin{array}{l}\text { Possible biological } \\
\text { function/pathological } \\
\text { process }\end{array}$ & Ref \\
\hline \multirow{2}{*}{ Atherosclerosis } & Human blood cells & RT-PCR & Upregulated & CircRNA0044073 & miRNA-107 & $\begin{array}{l}\text { JAK/STAT signaling } \\
\text { pathway }\end{array}$ & 27 \\
\hline & HUVECs & Microarray & Upregulated & CircRNA0003575 & $\begin{array}{l}\text { miR-199-3p, miR-9-5p, } \\
\text { miR-377-3p and miR-141- } \\
3 p\end{array}$ & Apoptosis pathway & 28 \\
\hline \multirow[t]{3}{*}{$\begin{array}{l}\text { Ischemic brain } \\
\text { injury }\end{array}$} & Mouse brain tissue & RT-PCR & Upregulated & CircTLK1 & miR-335-3p & $\begin{array}{l}\text { Inhibition of cell } \\
\text { differentiation }\end{array}$ & 32 \\
\hline & Mouse brain tissue & Microarray & Up-regulated & CircHectd1 & miR-142 & $\begin{array}{l}\text { Macroautophagy or } \\
\text { autophagy }\end{array}$ & 24 \\
\hline & Mice model & HTS & $40 \uparrow 47 \downarrow$ & $\begin{array}{l}\text { CircRNA17737, } \\
\text { CircRNA8828, } \\
\text { CircRNA14479, } \\
\text { CircRNA1059, } \\
\text { CircRNA9967, } \\
\text { CircRNA6952 }\end{array}$ & $\begin{array}{l}\mathrm{miR}-320-3 \mathrm{p} / \mathrm{miR}-873-5 \mathrm{p} / \\
\mathrm{miR}-351-3 \mathrm{p} ; \mathrm{miR}-665 / \\
\mathrm{miR}-127-5 \mathrm{p} / \mathrm{miR}-383-3 \mathrm{p} / \\
\mathrm{miR}-6315 / \mathrm{miR}-351-5 \mathrm{p} ; \\
\mathrm{miR}-3557-3 \mathrm{p} / \mathrm{miR}-351-5 \mathrm{p} / \\
\mathrm{miR}-298-5 \mathrm{p} / \mathrm{miR}-665 / \\
\mathrm{miR}-874-3 \mathrm{p} ; \mathrm{miR}-3541 / \\
\mathrm{miR}-204-3 \mathrm{p} / \mathrm{miR}-351-5 \mathrm{p} / \\
\mathrm{miR}-874-3 \mathrm{p} / \mathrm{miR}-343 ; \\
\mathrm{miR}-3099 / \mathrm{miR}-3584-5 \mathrm{p} / \\
\mathrm{miR}-3541 / \mathrm{miR}-3594-5 \mathrm{p} / \\
\mathrm{miR}-874-3 \mathrm{p}\end{array}$ & & 35 \\
\hline MMD & $\begin{array}{l}\text { Neutrophil } \\
\text { samples from } \\
\text { human }\end{array}$ & Microarray & $54 \uparrow 69 \downarrow$ & $\begin{array}{l}\text { CircRNA100146, } \\
\text { CircRNA102534, } \\
\text { CircRNA036592, } \\
\text { CircRNA405463, } \\
\text { CircRNA405324 }\end{array}$ & & & 40 \\
\hline \multirow[t]{2}{*}{ Others } & $\begin{array}{l}\mathrm{C} 57 \mathrm{BL} / 6 \mathrm{~J} \text { mice } \\
\text { model }\end{array}$ & Microarray & & $\begin{array}{l}\text { Circ006839 } \\
\text { Circ015350 } \\
\text { Circ000741 }\end{array}$ & $\begin{array}{l}\text { CRIM1 } \\
\text { NcoA2 } \\
\text { AKAP7 }\end{array}$ & $\begin{array}{l}\text { Proliferation and } \\
\text { apoptosis of vascular } \\
\text { endothelial cells }\end{array}$ & 5 \\
\hline & HT22 cells & Microarray & $3 \uparrow 12 \downarrow$ & CircRNA015947 & $\begin{array}{l}\text { miR-329-5p, miR-188-3p, } \\
\text { miR-5098, miR-3057-3p } \\
\text { and miR-683 }\end{array}$ & Apoptosis & 44 \\
\hline
\end{tabular}

CDK4, cyclin-dependent kinase 4; PES1, pescadillo ribosomal biogenesis factor 1; RT-PCR, reverse transcription-quantitative polymerase chain reaction assays; JAK1, Janus kinase 1; STAT3, signal transducer and activator of transcription 3; HUVECs, human umbilical vein endothelial cell; EndoMT, endothelialmesenchymal transition; HTS, high-throughput sequencing; CRIM1, cysteine-rich motor neuron 1 protein; circRNA, circular RNA; CVD, cerebrovascular disease; CVD, cerebral vascular disorder; miRNA, microRNA; circANRIL, circular antisense noncoding RNA in the INK4 locus; MMD, Moyamoya disease. 
ous studies reported that circRNAs bind such protein factors as RNA-pol II and Argonaute to regulate transcription in the transcription initiation region [16].

\section{Isolation of RNA-Binding Proteins}

CircRNAs that are expressed in the cytoplasm can bind proteins and inhibit their transport to the nucleus. Du et al. [21] found that circ-Foxo3 bound the aging-related proteins inhibitor of DNA binding 1 (ID1), E2F transcription factor 1 (E2F1), and hypoxia-inducible factor $1 \alpha(\mathrm{HIF} 1 \alpha)$ in the cytoplasm, preventing their nuclear localization and consequently leading to heart aging. Noncoding RNAs play an important role in the development and progression of many human diseases. Currently, research on the relationship between circRNAs and human diseases is mainly concentrated in the field of cancer [22], but progressively more circRNAs have been shown to play a role in the occurrence and development of CVDs [23] (Table 1). Below we will review the relationship between circRNAs and CVDs.

\section{CircRNAs and CVDs}

\section{CircRNAs and Atherosclerosis}

Atherosclerosis is the common pathological basis of many CVDs. Circular antisense noncoding RNA in the INK4 locus (circANRIL) is expressed in human vascular tissues, smooth muscle cells, monocytes, and macrophages and is involved in the development of atherosclerosis. Holdt et al. [24] provided strong evidence of an association between circANRIL and atherosclerosis. These authors suggested that circANRIL induces nucleolar stress and p53 activation, resulting in the induction of apoptosis and inhibition of proliferation, which are key cellular pathogenesis in atherosclerosis [24]. Other studies have shown that circANRIL may be involved in the development of atherosclerosis by regulating the expression of cyclin-dependent kinase 4 (CDK4) protein/variable reading frame inhibitor (INK4/ARF) [25]. Circ0044073 is another circRNA that is associated with atherosclerosis, which is confirmed in human specimens. Shen et al. [26] found that the expression of circ0044073 was upregulated and that the expression of miRNA-107 was downregulated in atherosclerotic blood cells. Circ0044073 can suppress the levels of miRNA-107 via a sponge mechanism. The tyrosine-protein kinase (JAK)/ signal transducer and activator of transcription (STAT) signaling pathway was activated in patients with athero-

Circular RNAs in Cerebral Vascular Disorders sclerosis, and circ0044073 activated the JAK/STAT signaling pathway in human vascular smooth muscle cells and endothelial cells [26]. Li et al. [27] found that circ0003575 was significantly upregulated in oxidized low-density lipoprotein-induced human umbilical vein endothelial cell (HUVECs). Silencing of circ0003575 promoted the proliferation and angiogenesis of HUVECs. High circ 0003575 levels were suggested to be involved in atherosclerosis after vascular injury [27]. Further studies of these circRNAs may reveal new therapeutic targets for the treatment of atherosclerosis.

\section{CircRNAs and Ischemic Brain Injury}

Ischemic brain injury is the main type of stroke, which is usually caused by cerebral thrombosis or embolism that leads to occlusion of the great arteries, resulting in loss of blood flow and oxygen supply to the affected area. Because of the very narrow therapeutic time window of intravenous recombinant tissue plasminogen activator treatment, new therapeutic strategies are needed. New drug interventions, including noncoding RNA strategies, have shown therapeutic promise [28]. Several studies have shown that noncoding RNAs are involved in poststroke repair and recovery, highlighting their potential therapeutic importance after stroke $[29,30]$. Wu et al. [31] showed that circRNA TLK1 (circTLK1) was significantly elevated in brain tissues in a mouse model of focal cerebral ischemia. CircTLK1 can act as an endogenous miRNA-335-3p sponge to inhibit its activity, resulting in an increase in the expression of 2,3,7,8-tetrachlorodibenzo-p-dioxin (TCDD)-inducible poly (ADP-ribose) polymerase and the subsequent exacerbation of neuronal injury (Fig. 1). The knockdown of circTLK1 significantly decreased infarct volume, attenuated neuronal injury, and improved neurological impairment. Plasma levels of circTLK1 also increased in acute ischemic stroke patients [31]. Han et al. [23] demonstrated that circHectd1 levels significantly increased in ischemic brain tissues in a mouse model. CircHectd 1 may serve as an endogenous miRNA-142 sponge to inhibit its activity, resulting in the inhibition of TCDD inducible poly (ADP-ribose) polymerase (TIPARP) expression and the subsequent inhibition of astrocyte activation via macroautophagy or autophagy. The knockdown of circHectd 1 expression significantly decreased infarct areas, attenuated neuronal deficits, and ameliorated astrocyte activation in ischemic brain tissues in a mouse model [23]. Another study by this research group showed that circDLGAP4 levels significantly decreased in the ischemic mouse brain. CircDLGAP4 may serve as an endogenous miRNA-143 


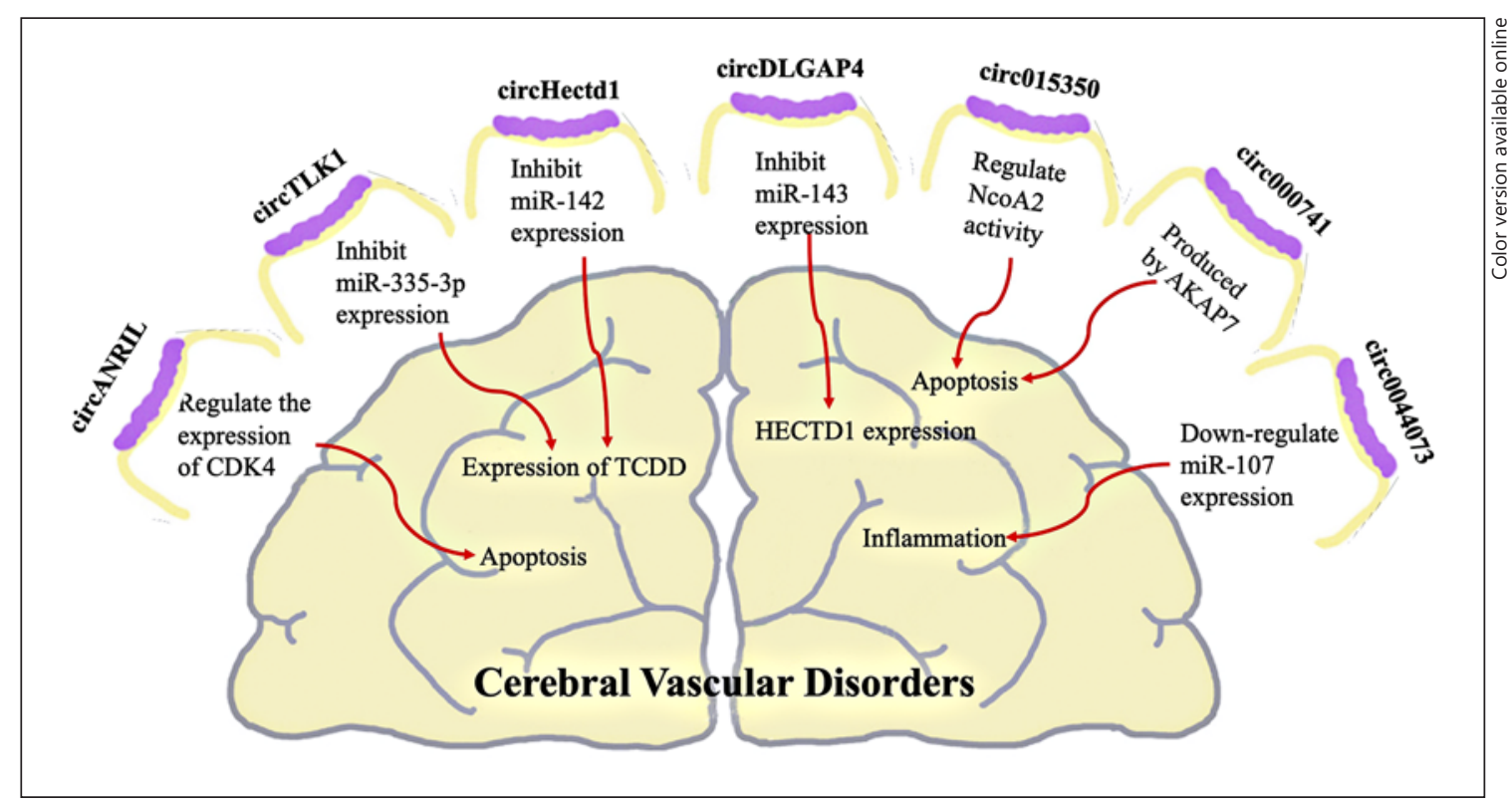

Fig. 1. Dysregulated circRNAs in CVD. TCDD, 2,3,7,8-tetrachlorodibenzo-p-dioxin; HECTD1, HECT domain E3 ubiquitin-protein ligase 1; circRNA, circular RNA; CVD, cerebral vascular disorder.

sponge that inhibits HECT domain E3 ubiquitin-protein ligase 1 (HECTD1) expression. The upregulation of circDLGAP4 expression significantly attenuated neurological impairment and decreased infarct volume and blood-brain barrier (BBB) damage [32].

The use of microarrays has identified a large number of circRNAs in models of cerebral ischemia-reperfusion injury. Liu et al. [33] screened the expression of circRNAs in a mouse model of cerebral ischemia and identified 1,027 differentially expressed circRNAs, including 914 upregulated and 113 downregulated circRNAs. Three circRNAs were further validated by quantitative real-time polymerase chain reaction (qRT-PCR), and bioinformatics analysis predicted that 15 miRNAs may interact with these circRNAs [33]. Duan et al. [34] identified 87 differentially expressed circRNAs (40 upregulated and 47 downregulated) by high-throughput sequencing in a rat model of middle cerebral artery occlusion. Six circRNAs were validated by qRT-PCR, and 23 miRNAs may interact with these circRNAs [34]. These results suggested that circRNAs may be novel diagnostic and therapeutic targets for stroke.

\section{CircRNAs and Moyamoya Disease}

Moyamoya disease (MMD) is a CVD that is characterized by chronic progressive stenosis or occlusion at the end of the bilateral internal carotid artery or anterior ce- rebral artery, beginning of the middle cerebral artery, and secondary abnormal vascular network in the basis cranii. The clinical manifestations of MMD are complex and variable, including cognitive impairment, epilepsy, and cerebral infarction [35]. Studies have shown that regulatory RNAs, such as long noncoding RNAs or miRNAs, play a role in promoting the development of MMD [36]. Compared with other types of miRNA sponges, circRNAs have more binding sites and higher expression levels and are thus considered more effective than linear RNAs in isolating miRNAs and regulating gene expression [37]. Zhao et al. [38] performed a circRNA microarray analysis of blood samples from patients with MMD and identified 146 differentially expressed circRNAs, including 29 that were upregulated and 117 that were downregulated. Five circRNAs (circRNA100914， circRNA103343， circRNA050898, circRNA101720, and circRNA067209) were confirmed by qRT-PCR [38]. In another circRNA microarray analysis of neutrophil samples from MMD patients, $\mathrm{Ma}$ et al. [39] identified 54 upregulated and 69 downregulated differentially expressed circRNAs. Five circRNAs (circRNA100146， circRNA102534， circRNA036592, circRNA405463, and circRNA405324) were validated by qRT-PCR [39]. These studies provide further insights into MMD and evidence that circRNAs may be novel biomarkers for the diagnosis of MMD. 
Other circRNAs Related to CVDs

Mehta et al. [5] reported that circ006839, a gene that was rich in cysteine-rich motor neuron 1 (CRIM1) protein, was differentially expressed at different time points of reperfusion after stroke and affected various pathophysiological processes after stroke. Studies have shown that CRIM1 gene defects influence the proliferation of vascular endothelial cells and promote endothelial cell apoptosis, suggesting that CRIM1-related circ006839 is associated with vascular dysfunction after stroke [40]. Circ015350 produced by the nuclear receptor coactivator 2 (NcoA2) gene was another circRNA investigated by Mehta et al. [5] Circ015350 was suggested to be related to apoptosis, oxidative stress, and other biological processes after stroke. NcoA2 is a transcription factor coactivator that can regulate the activity of transcription factors and inhibit the induction of HIF1 $\alpha$. The activity of NcoA2 affects tubule formation and the proliferation and development of vascular endothelial cells [41]. Circ015350 regulates the activity of transcription factors, providing a potential drug target for poststroke brain protection and treatment. Circ 000741 is produced by the protein kinase A-binding scaffolding molecule (AKAP7) gene and affects the expression of many transcription factors and apoptosis-related proteins after stroke [5]. AKAP7 is mainly expressed in lymphocytes, and its expression increases in peripheral blood after stroke and is closely related to BBB integrity. AKAP7-related circ000741 is expected to be a potential molecular marker in peripheral blood that may reflect the progression of stroke [42].

In a microarray study that utilized an oxygen-glucose deprivation/reoxygenation cell model, identified 15 circRNAs that were significantly differentially expressed (3 were upregulated, and 12 were downregulated). Circ015947 was further verified by qRT-PCR. Bioinformatics analysis predicted that circ 015947 may interact with miRNA-329-5p, miRNA-188-3p, miRNA-5098,
miRNA-3057-3p, and miRNA-683. This study showed that stroke may alter the expression of circRNAs, with possible functional implications for poststroke pathophysiology.

\section{Conclusions}

As a kind of noncoding RNA, circRNAs have become a focus of research in recent years because of their high stability and tissue specificity. Studies have shown that circRNAs are related to pathophysiological processes of many diseases, including inflammation, oxidative stress, mitochondrial dysfunction, and apoptosis. A large number of circRNAs are present in saliva, blood, and other secretions. They may be useful as biomarkers of disease diagnosis or prediction, especially the occurrence, development, and prognosis of CVDs. Findings from studies of the involvement of circRNAs in CVDs have provided a novel theoretical basis of the occurrence and development of CVDs and revealed new perspectives of poststroke brain protection.

\section{Conflict of Interest Statement}

The authors have no conflicts of interest to declare.

\section{Funding Sources}

The authors did not receive any funding.

\section{Author Contributions}

W.L. was responsible for the literature search and discussion. Y.G. revised it critically for important intellectual content and approved the final version.

\section{References}

1 Sanger HL, Klotz G, Riesner D, Gross HJ, Kleinschmidt AK. Viroids are single-stranded covalently closed circular RNA molecules existing as highly base-paired rod-like structures. Proc Natl Acad Sci USA. 1976 Nov; 73(11):3852-6

2 Salzman J, Gawad C, Wang PL, Lacayo N, Brown PO. Circular RNAs are the predominant transcript isoform from hundreds of human genes in diverse cell types. PloS One. 2012;7(2):e30733.
3 Salzman J, Chen RE, Olsen MN, Wang PL, Brown PO. Cell-type specific features of circular RNA expression. PLoS Genet. 2013; 9(9):e1003777.

4 Rybak-Wolf A, Stottmeister C, Glažar P, Jens $\mathrm{M}$, Pino N, Giusti S, et al. Circular RNAs in the mammalian brain are highly abundant, conserved, and dynamically expressed. Mol Cell. 2015 Jun 4;58(5):870-85.

5 Mehta SL, Pandi G, Vemuganti R. Circular RNA expression profiles alter significantly in mouse brain after transient focal ischemia. Stroke. 2017 Sep;48(9):2541-8.
6 Fan L, Gui L, Chai EQ, Wei CJ. Routine hematological parameters are associated with short- and long-term prognosis of patients with ischemic stroke. J Clin Lab Anal. 2018 Feb;32(2):e22244.

7 Jeck WR, Sorrentino JA, Wang K, Slevin MK, Burd CE, Liu J, et al. Circular RNAs are abundant, conserved, and associated with ALU repeats. RNA. 2013 Feb;19(2):141-57.

8 Zhang XO, Wang HB, Zhang Y, Lu X, Chen LL, Yang L. Complementary sequence-mediated exon circularization. Cell. 2014 Sep 25; 159(1):134-47.
Circular RNAs in Cerebral Vascular Disorders
Eur Neurol 2021;84:230-236 DOI: $10.1159 / 000515807$ 
9 Zhang Y, Zhang XO, Chen T, Xiang JF, Yin $\mathrm{QF}$, Xing YH, et al. Circular intronic long noncoding RNAs. Mol Cell. 2013 Sep 26; 51(6):792-806.

10 Conn SJ, Pillman KA, Toubia J, Conn VM, Salmanidis M, Phillips CA, et al. The RNA binding protein quaking regulates formation of circRNAs. Cell. 2015 Mar 12;160(6):112534.

11 Memczak S, Jens M, Elefsinioti A, Torti F, Krueger J, Rybak A, et al. Circular RNAs are a large class of animal RNAs with regulatory potency. Nature. 2013 Mar 21;495(7441): 333-8.

12 Li Z, Huang C, Bao C, Chen L, Lin M, Wang $\mathrm{X}$, et al. Exon-intron circular RNAs regulate transcription in the nucleus. Nat Struct Mol Biol. 2015 Mar;22(3):256-64.

13 Ashwal-Fluss R, Meyer M, Pamudurti NR, Ivanov $\mathrm{A}$, Bartok $\mathrm{O}$, Hanan $\mathrm{M}$, et al. circRNA biogenesis competes with pre-mRNA splicing. Mol Cell. 2014 Oct 2;56(1):55-66.

14 Salmena L, Poliseno L, Tay Y, Kats L, Pandolfi PP. A ceRNA hypothesis: the rosetta stone of a hidden RNA language? Cell. 2011 Aug 5;146(3):353-8.

15 Hansen TB, Jensen TI, Clausen BH, Bramsen JB, Finsen B, Damgaard CK, et al. Natural RNA circles function as efficient microRNA sponges. Nature. 2013 Mar 21;495(7441): 384-8.

16 Hansen TB, Wiklund ED, Bramsen JB, Villadsen SB, Statham AL, Clark SJ, et al. miRNA-dependent gene silencing involving Ago2-mediated cleavage of a circular antisense RNA. EMBO J. 2011 Sep 30;30(21): 4414-22.

17 Hou LD, Zhang J. Circular RNAs: an emerging type of RNA in cancer. Int J Immunopathol Pharmacol. 2017 Mar;30(1):1-6.

18 Meng X, LiX, Zhang P, Wang J, Zhou Y, Chen M. Circular RNA: an emerging key player in RNA world. Brief Bioinform. 2017 Jul 1;18(4): 547-57.

19 Chen CY, Sarnow P. Initiation of protein synthesis by the eukaryotic translational apparatus on circular RNAs. Science. 1995 Apr 21; 268(5209):415-7.

20 Yang Y, Fan X, Mao M, Song X, Wu P, Zhang $\mathrm{Y}$, et al. Extensive translation of circular RNAs driven by N(6)-methyladenosine. Cell research. 2017 May;27(5):626-41.
21 Du WW, Zhang C, Yang W, Yong T, Awan FM, Yang BB. Identifying and characterizing circRNA-protein interaction. Theranostics. 2017;7(17):4183-91

22 Patop IL, Kadener S. circRNAs in cancer. Curr Opin Genet Dev. 2018 Feb;48:121-7.

23 Han B, Zhang Y, Zhang Y, Bai Y, Chen X, Huang $R$, et al. Novel insight into circular RNA HECTD1 in astrocyte activation via autophagy by targeting MIR142-TIPARP: implications for cerebral ischemic stroke. Autophagy. 2018;14(7):1164-84.

24 Holdt LM, Stahringer A, Sass K, Pichler G, Kulak NA, Wilfert W, et al. Circular non-coding RNA ANRIL modulates ribosomal RNA maturation and atherosclerosis in humans. Nat Commun. 2016 Aug 19;7:12429.

25 Burd CE, Jeck WR, Liu Y, Sanoff HK, Wang $Z$, Sharpless NE. Expression of linear and novel circular forms of an INK4/ARF-associated non-coding RNA correlates with atherosclerosis risk. PLoS Genet. 2010 Dec 2;6(12): e1001233.

26 Shen L, Hu Y, Lou J, Yin S, Wang W, Wang $\mathrm{Y}$, et al. CircRNA0044073 is upregulated in atherosclerosis and increases the proliferation and invasion of cells by targeting miR107. Mol Med Rep. 2019 May;19(5):3923-32.

27 Li CY, Ma L, Yu B. Circular RNA hsa circ_0003575 regulates oxLDL induced vascular endothelial cells proliferation and angiogenesis. Biomed Pharmacother. 2017 Nov; 95:1514-9.

28 Kaur H, Sarmah D, Saraf J, Vats K, Kalia K, Borah A, et al. Noncoding RNAs in ischemic stroke: time to translate. Ann NY Acad Sci. 2018 Jun;1421(1):19-36.

29 Ouyang YB, Lu Y, Yue S, Xu LJ, Xiong XX, White RE, et al. miR-181 regulates GRP78 and influences outcome from cerebral ischemia in vitro and in vivo. Neurobiol Dis. 2012 Jan;45(1):555-63.

30 Zhang X, Tang X, Liu K, Hamblin MH, Yin KJ. Long noncoding RNA Malat1 regulates cerebrovascular pathologies in ischemic stroke. J Neurosci. 2017 Feb 15;37(7):1797806.

31 Wu F, Han B, Wu S, Yang L, Leng S, Li M, et al. Circular RNA TLK1 aggravates neuronal injury and neurological deficits after ischemic stroke via miR-335-3p/TIPARP. J. Neurosci. 2019 Jul 16;39(37):7369-93.
32 Bai Y, Zhang Y, Han B, Yang L, Chen X, Huang R, et al. Circular RNA DLGAP4 ameliorates ischemic stroke outcomes by targeting miR-143 to regulate endothelial-mesenchymal transition associated with bloodbrain barrier integrity. J Neurosci. 2018 Jan 3; 38(1):32-50.

33 Liu C, Zhang C, Yang J, Geng X, Du H, Ji X, et al. Screening circular RNA expression patterns following focal cerebral ischemia in mice. Oncotarget. 2017 Oct 17;8(49):8653547.

34 Duan X, Li L, Gan J, Peng C, Wang X, Chen $\mathrm{W}$, et al. Identification and functional analysis of circular RNAs induced in rats by middle cerebral artery occlusion. Gene. 2019 Jun 15; 701:139-45.

35 Fujimura M, Bang OY, Kim JS. Moyamoya disease. Front Neurol Neurosci. 2016;40:20420.

36 Gao F, Yu L, Zhang D, Zhang Y, Wang R, Zhao J. Long noncoding RNAs and their regulatory network: potential therapeutic targets for adult moyamoya disease. World Neurosurg. 2016 Sep;93:111-9.

37 Wilusz JE, Sharp PA. Molecular biology. A circuitous route to noncoding RNA. Science. 2013 Apr 26;340(6131):440-1.

38 Zhao M, Gao F, Zhang D, Wang S, Zhang Y, Wang R, et al. Altered expression of circular RNAs in moyamoya disease. J Neurol Sci. 2017 Oct 15;381:25-31.

$39 \mathrm{Ma}$ Q, Li L, Yu B, Jiao L, Han Z, Zhao H, et al. Circular RNA profiling of neutrophil transcriptome provides insights into asymptomatic Moyamoya disease. Brain Res. 2019 Sep 15;1719:104-12.

40 Nakashima Y, Takahashi S. Induction of cysteine-rich motor neuron 1 mRNA expression in vascular endothelial cells. Biochem Biophys Res Commun. 2014 Aug 22;451(2):2358.

41 Tsai CH, Li CH, Liao PL, Cheng YW, Lin CH, Huang SH, et al. NcoA2-dependent inhibition of HIF- $1 \alpha$ activation is regulated via AhR. Toxicol Sci. 2015 Dec;148(2):517-30.

42 O'Connell GC, Treadway MB, Petrone AB, Tennant CS, Lucke-Wold N, Chantler PD, et al. Peripheral blood AKAP7 expression as an early marker for lymphocyte-mediated poststroke blood brain barrier disruption. Sci Rep. 2017 Apr 26;7(1):1172. 